\title{
Mean transit time and mean residence time for linear diffusion-convection-reaction transport system
}

\author{
JACEK WANIEWSKI $+\neq \uparrow *$
}

\begin{abstract}
$\dagger$ Institute of Biocybernetics and Biomedical Engineering PAS, ul. Trojdena 4, 02-109 Warsaw, Poland $\ddagger$ Division of Baxter Novum K56, Department of Clinical Sciences, Karolinska Institutet, Stockholm, Sweden ๆ Interdisciplinary Centre for Mathematical and Computation Modelling, Warsaw University, ul. Pawinskiego 5a, Warsaw, Poland
\end{abstract}

(Received 22 May 2006; revised 13 September 2006; in final form 15 February 2007)

\begin{abstract}
Characteristic times for transport processes in biological systems may be evaluated as mean transit times (MTTs) (for transit states) or mean residence times (MRT) (for steady states). It is shown in a general framework of a (linear) reaction-diffusion-convection equation that these two times are related. Analytical formulas are also derived to calculate moments of exit time distribution using solutions for a stationary state of the system.
\end{abstract}

Keywords: Mean transit time; Linear diffusion; Transit states; Steady states; Exit time distribution function; Higher moments

\section{Introduction}

Transport processes in various structures are investigated by the injection of a bolus amount of a marker at the inlet to the system and the measurement of the injected marker fraction at the outlet from the system as a function of time. For example, physiological experiments are carried out for the whole isolated organs, in which exchange of water and solutes between blood and the tissue involves a complex network of membranes and distributed transport barriers [1-3]. Another system of this kind is applied for the penetration of infrared photons into biological tissues [4-6].

Mean transit time (MTT) is usually defined for experiments with a momentary input to the system of a known amount of substance, $M_{0}$ (often a small amount of a labelled form of the investigated substance), and the recording of its concentration, $C$, at the outlet from the system as a function of time. MTT may then be calculated as $\int_{0}^{\infty} t C(t) \mathrm{d} t / \int_{0}^{\infty} C(t) \mathrm{d} t[1]$. The theoretical evaluation of MTT needs a solution of the model equations for the system, which is generally described by ordinary differential equations (compartment systems) or partial differential equations (distributed systems). The transport may be convective, diffusive or combined. It was shown that for pure diffusive or pure convective transport in some simple transport systems this definition of MTT is equivalent to another one that is formulated for the steady state of the system [7-9]. In this case, for a steady input of the solute, the system reaches after some time a steady state of the concentration distribution and

\footnotetext{
*Email: jacekwan@ibib.waw.pl
} 
the constant output of the substance. Under these conditions one can calculate the mean residence time (MRT), as MRT $=M_{\mathrm{S}} / J_{\mathrm{Sin}}$, where $M_{\mathrm{S}}$ is the amount of the substance in the system and $J_{\operatorname{Sin}}$ is the rate of the substance input to the system. For pure convective transport and pure diffusive transport in the system, MRT is equal to MTT calculated for the transient passage of the substance through the system $[7,8]$.

The typical biological applications that involve the estimation of MTT are in medicine, physiology, cell biology and pharmacokinetics. The experiments with the in vivo perfused organs are based on the convective transport of the (labelled) solute to the organ by a bolus infusion to the organ artery and the measurements of the solute concentration in blood leaving the organ via its vein [1]. The transport of the solute from blood to the tissue and back may be diffusive or combined diffusive-convective. An example of such investigation may be found in the studies of the transport in the liver using, among other data, MTT and the variance of the distribution of transit times [3,10,11]. Another problem, diffusion of a substrate from the cell surface to the site inside the cell, where the enzyme that consumes the substrate is located, is a template for the discussion of MTT and MRT for the diffusive transport by Hardt [8].

The transport system involved in the peritoneal dialysis and the local drug delivery is more complex [12]. It comprises the transport (diffusive or diffusive-convective) through the organ surface and the distributed internal source or sink (depending on the direction of the solute transport, which may be regulated by imposing different boundary conditions) within the tissue. The flow through the surface and the capillary wall is in general bi-directional, but one may select some special boundary conditions which make the transport unidirectional, and in this way simplify the interpretation of the results. Furthermore, the combined diffusive and convective transport is typical for peritoneal dialysis, although for solutes of low molecular weight, diffusion is prevailing and one may approximately neglect the convective transport $[13,14]$. The status of microcirculation may also contribute to the efficiency of these therapies [1,15-17].

Transit times for infrared photons injected into the tissue, scattered there, and recorded at a different site on the tissue surface are used for obtaining information about the tissue and its microcirculation [4]. In particular, the first moment of the distribution of time of photon flights can be used for the analysis of changes in the absorptive characteristics of the tissue, and the inclusion of the second moment improves the analysis [5,6].

MTT, called also the mean first passage time, is usually defined for a system comprising a solute with total mass $M$, which may be a function of time, $t$ and the solute inflow, $F_{\text {in }}$, to the system and the solute outflow, $F_{\text {out }}$, from the system, defined at the inlet to and the outlet from the system, respectively, as functions of time. Under the assumptions: (1) initial load of mass $M_{0}$ at the inlet to the system, (2) no other solute inflow to the system, and (3) no back-flow of molecules that leave the system through the inlet, one defines the MTT, $\tau$, as $[1,8]$ :

$$
\tau=\int_{0}^{\infty} t h(t) \mathrm{d} t
$$

where $h(t)$ is the distribution of times for molecules to reach the exit from the system, and is given by the formula $[1,8]$ :

$$
h(t)=\frac{F_{\text {out }}(t)}{\int_{0}^{\infty} F_{\text {out }}(t) \mathrm{d} t}
$$


Taking into account that under the stated assumptions: $F_{\text {out }}(t)=-\mathrm{d} M(t) / \mathrm{d} t, M(t) \rightarrow 0$ for $t \rightarrow \infty$, and therefore $\int_{0}^{\infty} F_{\text {out }}(t) \mathrm{d} t=M_{0}$, one gets:

$$
h(t)=-\frac{1}{M_{0}} \frac{\mathrm{d} M}{\mathrm{~d} t}
$$

and, using formula (1):

$$
\tau=\frac{1}{M_{0}} \int_{0}^{\infty} M(t) \mathrm{d} t
$$

where it is assumed that $t M(t) \rightarrow 0$ if $t \rightarrow \infty$.

If the system is in the steady state then the solute concentration, $C_{\mathrm{S}}$, within the system, the solute mass, $M_{\mathrm{S}}$, in the system, and the solute inflow, $F_{\mathrm{Sin}}$, to the system are constant. The $\mathrm{MRT}, \tau_{\text {res }}$, i.e. the mean time the particle that enters the system spends in it, is given as [8]:

$$
\tau_{\text {res }}=\frac{M_{\mathrm{S}}}{F_{\text {Sin }}}
$$

The equality of $\tau$ and $\tau_{\text {res }}$ was initially shown for convective transport of a flow indicator for measurements of blood flow and volume [7]. Next, the Hardt theorem stated that for the systems with pure diffusion and the inflow and outflow only through the boundary, $\tau=\tau_{\text {res }}$ [8]. It was mentioned by Hardt that this theorem is also valid for the systems with the distributed source/sink of the solute [8]. We show that this result is valid for a general linear diffusion-convection-reaction system (described by elliptic differential operator), and that higher moments of the transit time distribution function may also be calculated using a steady state solution for the system.

\section{Formulation of the model}

The basic equation for $C(x, t)$ in a connected bounded region $V$ of $n$-dimensional Euclidian space $\mathbb{R}^{n}, n \geq 1$, is:

$$
\frac{\partial C}{\partial t}=L C+S
$$

where $L$ is a linear operator defined as

$$
L C=-\operatorname{div} \mathbf{F}-A C
$$

and

$$
\mathbf{F}=-\mathbf{D} \operatorname{grad} C+\mathbf{J} C
$$

for $\mathbf{D}=\left(D_{i j}\right)(x), \mathbf{J}=\left(J_{i}\right)(x), A=A(x) \geq 0$, and $S=S(x) \geq 0, i, j=1, \ldots \mathbf{n}$ and symbols in bold describe $\mathbf{n}$-dimensional vectors or $\mathbf{n} \times \mathbf{n}$ matrices. Furthermore, it is assumed that diffusivity matrix is symmetric, $D_{i j}=D_{j i}$, and that $\boldsymbol{\xi} \cdot \mathbf{D}(x) \xi \geq \theta \xi \xi$ for some $\theta>0$, almost all $x \in V$, and all $\xi \in \mathbb{R}^{n}$, where $\mathbb{R}^{n}$ is $n$-dimensional Euclidean space, and $\mathbb{R}=\mathbb{R}^{1}$ is the set of real numbers. The last condition states that $L$ is an elliptic operator, and guarantees that diffusion goes along the concentration gradient [18]. 
Boundary conditions: The boundary $\partial V$ of the region $V$ can be split in three parts $\partial V=\partial V_{W} \cup \partial V_{\mathrm{D}} \cup \partial V_{N}$, with the following characteristics:

(1) "no-flux" Robin boundary condition (impermeable wall) on $\partial V_{W}: F_{n}(x, t)=0$ for $x \in \partial V_{W}, t>0$,

(2) Dirichlet boundary condition for $\partial V_{\mathrm{D}}: C(x, t)=\varphi(x)$ for $x \in \partial V_{\mathrm{D}}, t>0$,

(3) Robin boundary condition for $\partial V_{N}: F_{n}(x, t)=-\psi(x)$ for $x \in \partial V_{N}, t>0$,

where subscript $n$ denotes the component normal to the boundary in the direction outward from $V$.

The third boundary condition, known also as the Dankwerts condition $[3,10,11]$, includes a practically important case of purely convective flow: $(\partial C / \partial n)(x, t)=0$ and $C(x, t)=\phi(x)$ for $x \in \partial V_{N}, t>0$.

Initial condition: $C(x, 0)=C_{0}(x)$ for $x \in V \partial V$.

The Green function $G(x, y, t)$ for equation (6) is defined, assuming $x$ as a parameter and $y$ and $t$ as variables, as the solution of the following equation for any $x \in V$

$$
\frac{\partial G}{\partial t}=L_{y}^{*} G
$$

where the linear operator $L_{y}^{*}$ is defined as

$$
L_{y}^{*} G=-\operatorname{div}\left(\mathbf{F}^{*}\right)-(\operatorname{div} \mathbf{J}+A) G
$$

and

$$
\mathbf{F}^{*}=-\mathbf{D} \operatorname{grad} G-\mathbf{J} G
$$

for the same parameters that appear in equation (6), and all spatial derivatives taken in variable $y$.

Boundary conditions for $G$ in variable $y$ for any $x \in V \backslash \partial V$ are:

(1) "no diffusive flux" Neumann boundary condition on $\partial V_{W} \cup \partial V_{N}:(\mathbf{D} \operatorname{grad} G)_{n}(x, y, t)$ $=0$ for $y \in \partial V_{W} \cup \partial V_{N}, t>0$,

(2) Dirichlet boundary condition for $\partial V_{\mathrm{D}}: G(x, y, t)=0$ for $y \in \partial V_{\mathrm{D}}, t>0$,

Initial condition for $G: G(x, y, 0)=\delta(x-y)$ for $x$ and $y$ in $V \partial V$.

One can show that $C$ can be expressed by $G, S, \varphi$ and $\psi$, and the initial solution as follows:

$$
\begin{aligned}
C(x, t)= & \int_{V} G(x, y, t-s) C(y, s) \mathrm{d} y+\int_{S}^{t} \int_{V} G(x, y, t-u) S(y) \mathrm{d} y \mathrm{~d} u \\
& -\int_{s}^{t} \int_{\partial V_{\mathrm{D}}}(\mathbf{D}(y) \operatorname{grad} G(x, y, t-u))_{n} \varphi(y) \mathrm{d} y \mathrm{~d} u \\
& -\int_{S}^{t} \int_{\partial V_{N}} G(x, y, t-u) \psi(y) \mathrm{d} y \mathrm{~d} u
\end{aligned}
$$

where $s \in[0, t]$. 
To proof formula (12) let us define

$$
\begin{aligned}
\mathrm{A}(x, t)= & \int_{S}^{t} \int_{V} G(x, y, t-u)\left(\frac{\partial C}{\partial u}-L_{y} C\right)(y, u)+\left(\frac{\partial G}{\partial u}+L_{y}^{*} G\right)(x, y, t-u) \\
& C(y, u) \mathrm{d} y \mathrm{~d} u
\end{aligned}
$$

Because

$$
\frac{\partial G}{\partial u}(x, y, t-u)=-\frac{\partial G}{\partial t}(x, y, t-u)=-L_{y}^{*} G(x, y, t-u)
$$

then, using equation (6),

$$
\mathrm{A}(x, t)=\int_{s}^{t} \int_{V} G(x, y, t-u) S(y) \mathrm{d} y \mathrm{~d} u
$$

On the other hand, equation (13) may be rearranged as follows:

$$
\begin{aligned}
\mathrm{A}(x, t)= & C(x, t)-\int_{V} G(x, y, t-s) C(y, s) \mathrm{d} y+\int_{s}^{t} \int_{\partial V} G(x, y, t-u) \mathbf{F}_{n}(y, u) \mathrm{d} y \mathrm{~d} u \\
& +\int_{s}^{t} \int_{\partial V}(\mathbf{D}(y) \operatorname{grad} G(x, y, t-u))_{n} C(y, u) \mathrm{d} y \mathrm{~d} u
\end{aligned}
$$

using the Gauss-Ostrogradzki theorem $\int_{V} \operatorname{div} \mathbf{B} \mathrm{d} y=\int_{\partial V} \mathbf{B}_{n} \mathrm{~d} y$, and the identity $\operatorname{div}(A \mathbf{B})=$ $A \operatorname{div} \mathbf{B}+\mathbf{B} \cdot \operatorname{grad} A$ for scalar function $A(y)$ and vector function $\mathbf{B}(y)$. The application of the boundary conditions for $C$ to the integrals over $\partial V$ in equation (15) and the comparison of equations (14) and (15) yield formula (12).

\section{Mean transit times for impulse inputs}

We assume that the system has a (stable) steady state $C_{\infty}(x)$ with total mass $M_{\infty}=\int_{V} C_{\infty}(x) \mathrm{d} x$, and this state is disturbed at $t=0$ by a pulse function concentrated at $x=z: M_{0} \delta(x-z) \delta(t)$. Let $C(x, t ; z)$ be the solution of equation (6) with the initial condition $C_{0}(x ; z)=C_{\infty}(x)+M_{0} \delta(x-z)$, and denote $M(z, t)=\int_{V} C(x, t ; z) \mathrm{d} x$. The MTT is defined as (c.f. equation (4))

$$
\tau(z)=-\frac{1}{M_{0}} \int_{0}^{\infty} t \mathrm{~d} m(z, t)
$$

where $m(z, t)=M(z, t)-M_{\infty}$.

Integrating by parts one gets

$$
\tau(z)=\frac{1}{M_{0}}\left[-\left.(\operatorname{tm}(z, t))\right|_{0} ^{\infty}+\int_{0}^{\infty} m(z, t) \mathrm{d} t\right]
$$


and, assuming that $\operatorname{tm}(z, t) \rightarrow 0$ for $t \rightarrow \infty$ and $z \in V \backslash\left\{\partial V_{W} \cap \partial V_{\mathrm{D}}\right\}$,

$$
\tau(z)=\frac{1}{M_{0}} \int_{0}^{\infty} m(z, t) \mathrm{d} t=\frac{1}{M_{0}} \int_{0}^{\infty} \int_{V}\left(C(x, t ; z)-C_{\infty}(x)\right) \mathrm{d} t \mathrm{~d} x .
$$

The solution $C(x, t ; z)$ may be found using formula (12) for the initial state $C_{0}(x ; z)$

$$
\begin{aligned}
C(x, t ; z)= & M_{0} G(x, z, t)+\int_{V} G(x, y, t-s) C_{\infty}(y) \mathrm{d} y+\int_{S}^{t} \int_{V} G(x, y, t-u) S(y) \mathrm{d} y \mathrm{~d} u \\
& -\int_{S}^{t} \int_{\partial V_{\mathrm{D}}}(\mathbf{D}(y) \operatorname{grad} G(x, y, t-u))_{n} \varphi(y) \mathrm{d} y \mathrm{~d} u \\
& -\int_{S}^{t} \int_{\partial V_{N}} G(x, y, t-u) \psi(y) \mathrm{d} y \mathrm{~d} u
\end{aligned}
$$

for any $s \in[0, t]$.

Note, that formula (12) applied for the steady state $C_{\infty}(x)$ implies that the terms with integrals in equation (19) sum up to $C_{\infty}(x)$. Thus

$$
C(x, t ; z)=M_{0} G(x, z, t)+C_{\infty}(x)
$$

and

$$
m(z, t)=M_{0} \int_{V} G(x, z, t) \mathrm{d} x
$$

From equation (9):

$$
\frac{\partial m}{\partial t}(z, t)=\left(L_{z}^{*} m\right)(z, t)
$$

with

(1) "no diffusive flux" Neumann boundary condition on $\partial V_{W} \cup \partial V_{N}:(\mathbf{D} \operatorname{grad} m)_{n}(z, t)=0$ for $z \in \partial V_{W} \cup \partial V_{N}, t>0$,

(2) Dirichlet boundary condition for $\partial V_{\mathrm{D}}: m(z, t)=0$ for $z \in \partial V_{\mathrm{D}}, t>0$,

(3) Initial condition for $m: m(z, 0)=M_{0}$ for $z$ in $V \backslash \partial V$.

From equations (18) and (20) it follows that

$$
\tau(z)=\int_{0}^{\infty} \int_{V} G(x, z, t) \mathrm{d} t \mathrm{~d} x
$$

Now, we may formulate an ordinary differential equation for $\tau(z)$. Let us consider

$$
\begin{aligned}
L_{z}^{*} \tau(z) & =\int_{0}^{\infty} \int_{V}\left(L_{z}^{*} G\right)(x, z, t) \mathrm{d} t \mathrm{~d} x=\int_{0}^{\infty} \int_{V} \frac{\partial G}{\partial t}(x, z, t) \mathrm{d} t \mathrm{~d} x \\
& =\int_{V}[G(x, z, \infty)-G(x, z, 0)] \mathrm{d} x
\end{aligned}
$$


Note, that $G(x, z, t) \rightarrow 0$ for $t \rightarrow \infty$, because of the boundary conditions for $G$, and that $G(x, z, 0)=\delta(x-z)$. Therefore

$$
\left(L_{z}^{*} \tau\right)(z)=-1
$$

This PDE is supplemented by the boundary conditions that are of the same type as for $G(x, z, t)$ :

(1) $(\partial \tau / \partial n)(z)=0$ for $z \in \partial V_{W} \cup \partial V_{N}$,

(2) $\tau(z)=0$ for $z \in \partial V_{\mathrm{D}}$.

Remark. Defining $\tau(z, t)=\int_{0}^{t} \int_{V} G(x, z, u) \mathrm{d} u \mathrm{~d} x$ one can derive an equation for $\tau(z, t)$ :

$$
\frac{\partial \tau}{\partial t}(z, t)=\left(L_{z}^{*} \tau\right)(z, t)+1
$$

\section{Mean residence time}

Let $\bar{C}(x)$ be a steady state solution for equation (6) for some boundary conditions, perhaps different from those already specified, which will be defined later on. Then, by equation (25),

$$
\int_{V} \bar{C}(z)\left(L_{z}^{*} \tau\right)(z) \mathrm{d} z=-\bar{M}
$$

where

$$
\bar{M}=\int_{V} \bar{C}(z) \mathrm{d} z
$$

On the other hand,

$$
\int_{V} \bar{C}(z)\left(L_{z}^{*} \tau\right)(z) \mathrm{d} z=\int_{V}\left(L_{z} \bar{C}\right)(z) \tau(z) \mathrm{d} z+\int_{\partial V}\left(\bar{C}(\mathbf{D} \operatorname{grad} \tau)_{n}+\tau \bar{F}_{n}\right)(z) \mathrm{d} z
$$

Because $\left(L_{z} \bar{C}\right)(z)=-S(z)$ by the definition of the stationary state, one gets, comparing equations (27) and (29) and using the boundary conditions for $\tau$,

$$
\bar{M}=-\int_{\partial V_{\mathrm{D}}}\left(\bar{C}(\mathbf{D} \operatorname{grad} \tau)_{n}\right)(z) \mathrm{d} z-\int_{\partial V_{N}}\left(\tau \overline{\mathbf{F}}_{n}\right)(z) \mathrm{d} z+\int_{V} S(z) \tau(z) \mathrm{d} z
$$

Let us assume now the boundary conditions for $\bar{C}$, c.f. [8],

(1) $\bar{F}_{n}(z)=0$ for $z \in \partial V_{W}$,

(2) $\bar{F}_{n}(z)=-\bar{\psi}(z), \bar{\psi}(z) \geq 0$ for $z \in \partial V_{N}$, with $\partial V_{N}$ considered as the inlet to the system,

(3) $\bar{C}(z)=\bar{\varphi}(z), \bar{\varphi}(z) \geq 0$ for $z \in \partial V_{\mathrm{D}}$, with $\partial V_{\mathrm{D}}$ considered as the outlet from the system.

The total inflow through the boundary, $\bar{F}_{\text {inlet }}$, is then

$$
\bar{F}_{\text {inlet }}=\int_{\partial V_{N}} \bar{\psi}(z) \mathrm{d} z
$$


The inflow-averaged mean time $\tau_{\text {inlet }}$ for the solute entering the system through $\partial V_{N}$ is defined as

$$
\tau_{\text {inlet }}=\frac{1}{\bar{F}_{\text {inlet }}} \int_{\partial V_{N}}(\tau \bar{\psi})(z) \mathrm{d} z
$$

The total inflow from the source is $F_{\text {source }}=\int_{V} S(z) \mathrm{d} z$, and the inflow-averaged mean time $\tau_{\text {source }}$ for the solute entering the system from the source is defined as

$$
\tau_{\text {source }}=\frac{1}{F_{\text {source }}} \int_{V}(\tau S)(z) \mathrm{d} z
$$

Equations (30)-(33) together with the boundary conditions for $\bar{C}$ yield the formula

$$
\tau_{\text {inflow }}-\int_{\partial V_{\mathrm{D}}}\left(\bar{\varphi}(\mathbf{D} \operatorname{grad} \tau)_{n}\right)(z) \mathrm{d} z=\frac{\bar{M}}{\bar{F}_{\text {inlet }}+F_{\text {source }}}
$$

where $\bar{M}$ and $\bar{F}_{\text {inlet }}$ are calculated for the steady state $\bar{C}$, and $\tau_{\text {inflow }}$ is defined as

$$
\tau_{\text {inflow }}=\frac{\bar{F}_{\text {inlet }}}{\bar{F}_{\text {inlet }}+F_{\text {source }}} \tau_{\text {inlet }}+\frac{F_{\text {source }}}{\bar{F}_{\text {inlet }}+F_{\text {source }}} \tau_{\text {source }} .
$$

Note that the residence time, $\tau_{\text {res }}$, for this system is defined as

$$
\tau_{\text {res }}=\frac{\bar{M}}{\bar{F}_{\text {inlet }}+F_{\text {source }}}
$$

c.f. equation (5). Thus, for $\bar{\varphi} \equiv 0$ one gets from equation (34) that

$$
\tau_{\text {inflow }}=\tau_{\text {res }}
$$

i.e. the Hardt theorem [8]. Formula (34) may be therefore considered as a generalization of the Hardt theorem to arbitrary Dirichlet conditions at the outlet from the system.

The inflow-averaged mean time $\tau_{\text {inflow }}$ for the solute entering the system through $\partial V_{N}$ depends on the boundary condition $\bar{\psi}(z)$ at the inlet. However, if $\bar{\psi}(z)$ does not depend on $z \in \partial V_{N}$, then

$$
\tau_{\text {inflow }}=\frac{1}{\left|\partial V_{N}\right|} \int_{\partial V_{N}} \tau(z) \mathrm{d} z
$$

$\tau_{\text {inflow }}$, given by equation (38), may be calculated directly if equation (25) is solved.

\section{Higher moments of exit function}

Using similar methods we can derive recursive equations and formulas for higher moments of the exit time distribution function defined as

$$
\tau_{k}(z)=-\frac{1}{M_{0}} \int_{0}^{\infty} t^{k} \mathrm{~d} m(t ; z)
$$


for integer $k \geq 0$. Note that $\tau_{0}(z)=1$. In the following, we assume that $t^{k} m(t ; z) \rightarrow 0$ and $t^{k-1} G(x, z, t) \rightarrow 0$ for $t \rightarrow \infty, z \in V \backslash\left\{\partial V_{W} \cap \partial V_{\mathrm{D}}\right\}$, and all integer $k \geq 1$. Then, for $k \geq 1$

$$
\tau_{k}(z)=\frac{k}{M_{0}} \int_{0}^{\infty} t^{k-1} m(t ; z) \mathrm{d} t=k \int_{0}^{\infty} \int_{V} t^{k-1} G(x, z, t) \mathrm{d} t \mathrm{~d} x
$$

c.f. equations (17) and (23) and their derivations. The PDE for $\tau_{k}(z)$ is obtained in the same way as equation (25):

$$
\left(L_{z}^{*} \tau_{k}\right)(z)=-k \tau_{k-1}(z)
$$

and the boundary conditions are the same as those for equation (25).

The theorem by Hardt about the description of MTT by MRT [8], section 4, may be generalized for higher moments of exit functions. Let us define the following parameters:

$$
\begin{gathered}
\tau_{k, \text { source }}=\frac{1}{F_{\text {source }}} \int_{V}\left(\tau_{k} S\right)(z) \mathrm{d} z \\
\tau_{k, \text { inlet }}=\frac{1}{\bar{F}_{\text {inlet }}} \int_{\partial V_{N}}\left(\tau_{k} \bar{\psi}\right)(z) \mathrm{d} z \\
\bar{\tau}_{k}=\frac{1}{\bar{M}} \int_{V}\left(\bar{C} \tau_{k}\right)(z) \mathrm{d} z
\end{gathered}
$$

for the steady state $\bar{C}$. Then

$$
\tau_{k, \text { inflow }}-\int_{\partial V_{\mathrm{D}}}\left(\bar{\varphi}\left(\mathbf{D} \operatorname{grad} \tau_{k}\right)_{n}\right)(z)=\frac{k \bar{M} \bar{\tau}_{k-1}}{\bar{F}_{\text {inlet }}+F_{\text {source }}}
$$

where $\bar{M}$ and $\bar{F}_{\text {inlet }}$ are calculated for the steady state $\bar{C}$, and $\tau_{\mathrm{k} \text {,inflow }}$ is defined as

$$
\tau_{k, \text { inflow }}=\frac{\bar{F}_{\text {inlet }}}{\bar{F}_{\text {inlet }}+F_{\text {source }}} \tau_{k \text {,inlet }}+\frac{F_{\text {source }}}{\bar{F}_{\text {inlet }}+F_{\text {source }}} \tau_{k \text {,source }}
$$

For $k=1$, formulae (42)-(46) reduce to those given in section 4 .

\section{Examples}

Let us consider the transport equation with constant coefficients on interval $[0, L]$

$$
\frac{\partial C}{\partial T}=D \frac{\partial^{2} C}{\partial X^{2}}-J \frac{\partial C}{\partial X}-A C+S
$$

After rescaling with $x=X / L$ and $t=D T / L^{2}$ we get

$$
\frac{\partial C}{\partial t}=\frac{\partial^{2} C}{\partial x^{2}}-j \frac{\partial C}{\partial x}-a C+s
$$


with the coefficients $j=J L / D, a=A L^{2} / D, s=S L^{2} / D$. Equation (25) is now

$$
\frac{\partial^{2} \tau}{\partial z^{2}}+j \frac{\partial \tau}{\partial z}-a \tau=-1
$$

with $(\partial \tau / \partial z)(0)=0$ and $\tau(1)=0$. The solution of equation (49) for $j, a \neq 0$ is

$$
\tau(z)=\frac{1}{a}\left(1-\exp (j(1-z) / 2) \frac{u \cosh (u z / 2)+j \sinh (u z / 2)}{u \cosh (u / 2)+j \sinh (u / 2)}\right)
$$

where $u=\sqrt{j^{2}+4 a}$.

Important special cases of solution (50) are

(1) for $j \neq 0, a=0$

$$
\tau(z)=\frac{1}{j}\left(1-z-\frac{\exp (-j)}{j}(\exp (j(1-z))-1)\right)
$$

(2) for $j=0, a>0$

$$
\tau(z)=\frac{1}{a}\left(1-\frac{\cosh (\sqrt{a} z)}{\cosh (\sqrt{a})}\right)
$$

(3) for $j=0, a=0$

$$
\begin{gathered}
\tau(z)=\frac{1}{2}\left(1-z^{2}\right) \\
\tau_{2}(z)=\frac{1}{2}\left(\frac{5}{6}-z^{2}\left(1-\frac{z^{2}}{6}\right)\right)
\end{gathered}
$$

and, for

$$
\begin{gathered}
\operatorname{var}(z)=-\frac{1}{M_{0}} \int_{0}^{\infty}(t-\tau)^{2} \mathrm{~d} m(t ; z)=\tau_{2}-\tau^{2}, \\
\operatorname{var}(z)=\frac{1}{6}\left(1-z^{4}\right) .
\end{gathered}
$$

For the Dirichlet boundary conditions at both boundaries for the main equation (49) one has the boundary conditions for $\tau: \tau(0)=0$ and $\tau(1)=0$. Then, the solution of equation (49) for $j, a \neq 0$ is

$$
\tau(z)=\frac{1}{a}\left(1-\mathrm{e}^{(u-j) z / 2}+\left(\mathrm{e}^{u / 2}-\mathrm{e}^{j / 2}\right) \mathrm{e}^{-j z / 2} \frac{\sinh (u z / 2)}{\sinh (u / 2)}\right)
$$

where $u=\sqrt{j^{2}+4 a}$. 
Some special cases of solution (56) are:

(1) for $j \neq 0, a=0$

$$
\tau(z)=\frac{1}{j}\left(\frac{1-\exp (-j z)}{1-\exp (-j)}-z\right)
$$

(2) for $j=0, a>0$

$$
\tau(z)=\frac{1}{a}\left(1-\exp (\sqrt{a} z)+(\exp (\sqrt{a})-1) \frac{\sinh (\sqrt{a} z)}{\sinh (\sqrt{a})}\right)
$$

(3) for $j=0, a=0$

$$
\tau(z)=\frac{1}{2} z(1-z)
$$

For the Neumann boundary conditions at both boundaries for the main equation (49) one has the boundary conditions for $\tau:(\mathrm{d} \tau / \mathrm{d} z)(0)=0$ and $(\mathrm{d} \tau / \mathrm{d} z)(1)=0$. Then, the solution of equation (49) for $a \neq 0$ is

$$
\tau(z)=\frac{1}{a}
$$

However, there is no solution if $a=0$.

\section{Discussion}

The equivalence of the passage times for transient and steady linear transport processes is shown for the general linear reaction-diffusion-convection equation in $\mathbb{R}^{n}, n>1$. It was described previously as the identity of MTT and MRT for pure convective and pure diffusive transport separately $[7,8]$. This identity is now proved for combined diffusive-convective transport with a possible linear chemical reaction. However, the "reaction" term may also describe an internal sink of the solute, extending considerably (e.g. due to the presence of blood microvessels and lymphatics) the range of systems under consideration. Furthermore, formula (46) demonstrates how higher moments of the distribution of passage times may be calculated from a steady state solution of the transport equation. The presented proof is based on the theory of linear parabolic differential equations, but physical arguments may refer to the assumption that the motion of any single molecule is independent of the motion of all other particles, as reflected by the linearity of the system. Therefore, the proof might be also based on the theory of stochastic processes, as it was used in Ref. [8].

Although the transport-reaction systems are in general non-linear, the linear equations are sometimes applicable for some of these systems. There may be several reasons for usefulness of linear systems in biological applications. First of all, some solutes are chemically "inert" and transported only by passive diffusion and convection, as for example, urea and creatinine, the basic markers for the clinical status of uremic patients and the efficiency of dialysis procedures, inert gases and some drugs, etc. [1,3,12,14-16]. Also, linear approximations for some general models are found to be accurate and practically useful descriptions, as in near-infrared 
spectroscopy [4-6]. Linearized systems appear also if small quantities of labelled markers are applied in experiments, because such small quantities do not change the state of the system [1]. Finally, linear systems are studied just as an approximation to obtain an initial insight into the system characteristics $[11,14]$. The parameters estimated from the experimental data for linear systems may later be used directly or by interpolation for solutes that are involved in, say, linear transport and non-linear reaction, etc. Note also that transit and residence times may be defined in a dose/concentration independent manner only for linear systems.

The theorem about the equality of MRT and MTT depends on the assumption that after perturbation of the system the total solute mass in the system returns to its steady state level asymptotically faster than $t^{-1}$, see equations (4) and (17)-(18). Furthermore, the derived formulas for higher moments of the exit function are based on the assumption that the asymptotic rate of return of the system mass to its steady state value is faster than $t^{-k}$ if the $k$ moment is considered (see section 5). These properties of the exit function, taken for granted in previous studies [7,8], are difficult to prove for general elliptic operators. The problem may be reduced to the investigation of the principal (maximal) eigenvalue of the elliptic operator that describes the system. Operator $L$, Equation (7), is in general the non-symmetric if convective term $\mathbf{J} \neq 0$. Nevertheless, it may often be shown that its principal eigenvalue is real and strictly negative and all other eigenvalues have real parts not higher than the principal eigenvalue if $A>0$, see a proof for the case of Dirichlet boundary condition in Ref. [18]. From such a characteristic of the operator's spectrum a conclusion about the desired asymptotic properties of the solute mass may easily be obtained.

The observation that information about the exit function for the linear transport systems may be derived at large extent from a steady state solution may be helpful in obtaining analytical formulas for systems with simple geometry, as shown in section 6 and Ref. [8]. It may potentially be useful for numerical analysis of the transport systems. It also allows for the derivation of some conclusions about transit characteristics from the studies of the system in the steady state.

\section{References}

[1] Bassingthwaighte, J.B. and Goresky, C.A., 1984, Modeling in the analysis of solute and water exchange in the microvasculature. In: S.R. Geiger, C.C. Michel, J.R. Pappenheimer and E.M. Renkin (Eds.) Handbook of Physiology_The Cardiovascular System IV, Vol. 4 (Microcirculation. Bethesda, MD: American Physiological Society).

[2] Beard, D.A. and Bassingthwaighte, J.B., 2000, Advection and diffusion of substances in biological tissues with complex vascular networks, Annals of Biomedical Engineering, 28(3), 253-268.

[3] Roberts, M.S. and Anissimov, Y.G., 1999, Modeling of hepatic elimination and organ distribution kinetics with the extended convection-dispersion model, Journal of Pharmacokinetics and Biopharmaceutics, 27(4), $343-382$.

[4] Arridge, S.R. and Schweiger, M., 1995, Direct calculation of the moments of the distribution of photon time of flight in the tissue with finite element method, Applied Optics, 34(15), 2683-2687.

[5] Liebert, A., Wabnitz, H., Grosenick, D., Moller, M., Macdonald, R. and Rinneberg, H., 2003, Evaluation of optical properties of highly scattering media by moments of distributions of times of flight of photons, Applied Optics, 42(28), 5785-5792.

[6] Liebert, A., Wabnitz, H., Steinbrink, J., Obrig, H., Moller, M., Macdonald, R., et al., 2004, Time-resolved multidistance near-infrared spectroscopy of the adult head: intracerebal and extracerebal absorption changes from moments of distribution of flight of photons, Applied Optics, 43(15), 3037-3047.

[7] Meier, P. and Zierler, K., 1954, On the theory of the indicator-dilution method for measurement of blood flow and volume, Journal of Applied Physiology, 12(6), 731-744.

[8] Hardt, S.L., 1981, The diffusion transit time; a simple derivation, Bulletin of Mathematical Biology, 45, 89-99.

[9] Segel, L.A., 1980, Mathematical Models in Molecular and Cellular Biology (Cambridge: Cambridge University Press) 
[10] Mellick, G.D., Anissimov, Y.G., Bracken, A.J. and Roberts, M.S., 1997, Metabolite mean transit times in the liver as predicted by various models of hepatic elimination, Journal of Pharmacokinetics and Biopharmaceutics, 25(4), 477-505.

[11] Roberts, M.S. and Rowland, M., 1986, A dispersion model of hepatic elimination: 1. Formulation of the model and bolus considerations, Journal of Pharmacokinetics and Biopharmaceutics, 14(3), 227-260.

[12] Flessner, M.F., 2005, The transport barrier in intraperitoneal therapy, American Journal of Physiology Renal Physiology, 288(3), F433-F442.

[13] Flessner, M.F., 2001, Transport of protein in the abdominal wall during intraperitoneal therapy. I. Theoretical approach, American Journal of Physiology. Gastrointestinal and Liver Physiology, 281(2), G424-G437.

[14] Waniewski, J., 2001, Physiological interpretation of solute transport parameters for peritoneal dialysis, Journal of Theoretical Medicine, 3, 177-190.

[15] Waniewski, J., Werynski, A. and Lindholm, B., 1999, Effect of blood perfusion on diffusive transport in peritoneal dialysis, Kidney International, 56(2), 707-713.

[16] Waniewski, J., 2002, Distributed modeling of diffusive solute transport in peritoneal dialysis, Annals of Biomedical Engineering, 30(9), 1181-1195.

[17] Waniewski, J., 2004, A mathematical model of local stimulation of perfusion by vasoactive agent diffusing from tissue surface, Cardiovascular Engineering An International Journal, 4(1), 115-123.

[18] Evans, L.C., 1998, Partial Differential Equations (Providence, RI: American Mathematical Society). 


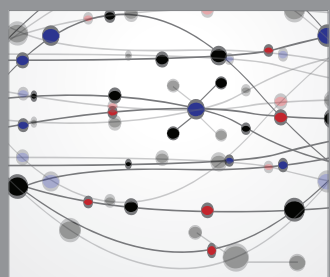

The Scientific World Journal
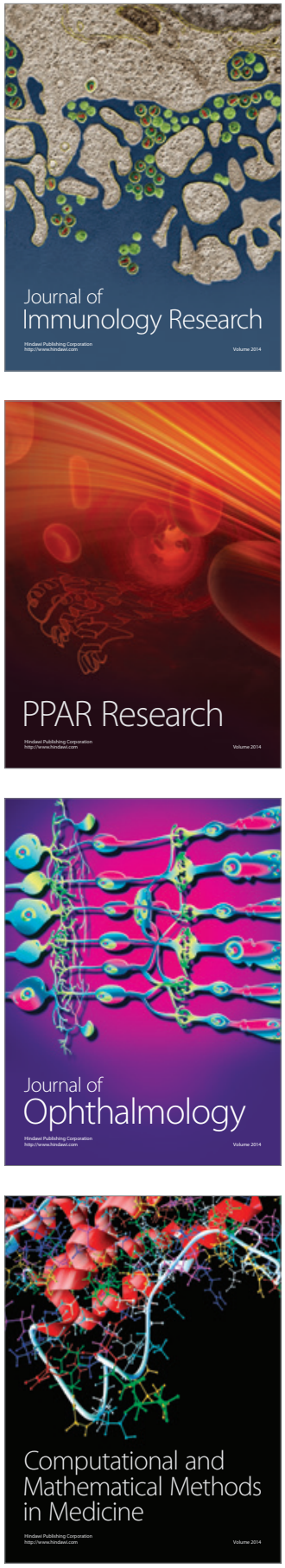

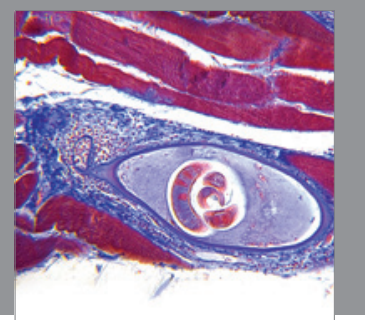

Gastroenterology

Research and Practice
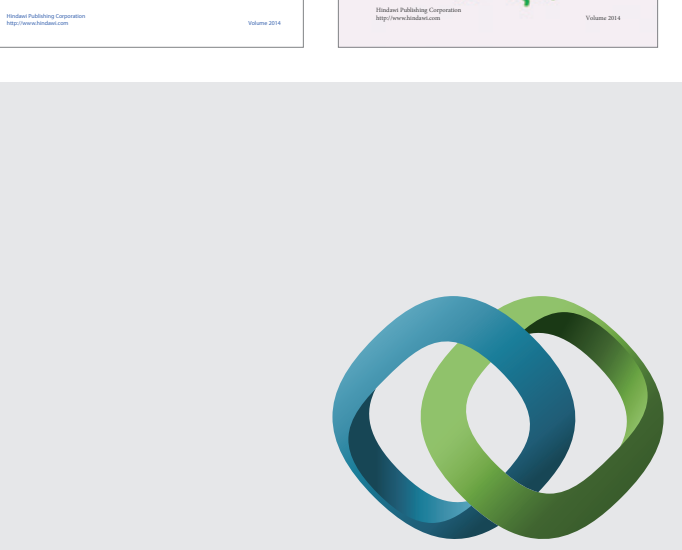

\section{Hindawi}

Submit your manuscripts at

http://www.hindawi.com
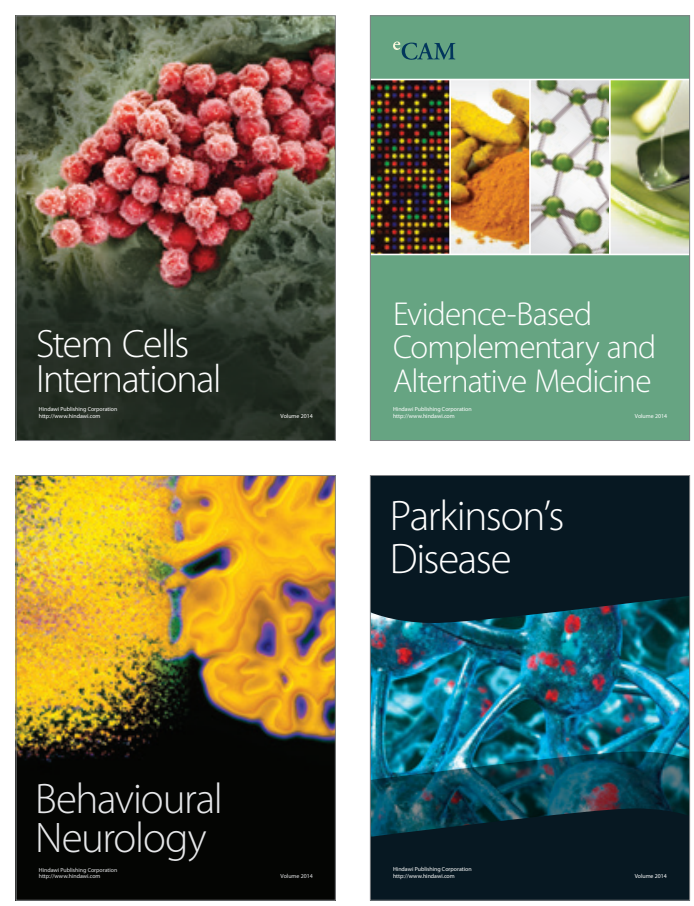

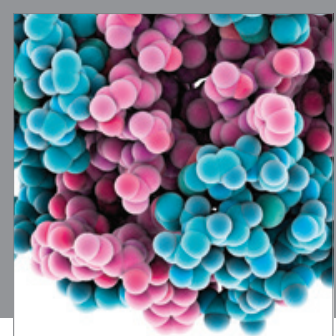

Journal of
Diabetes Research

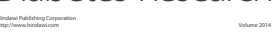

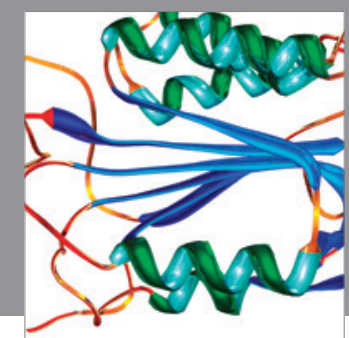

Disease Markers
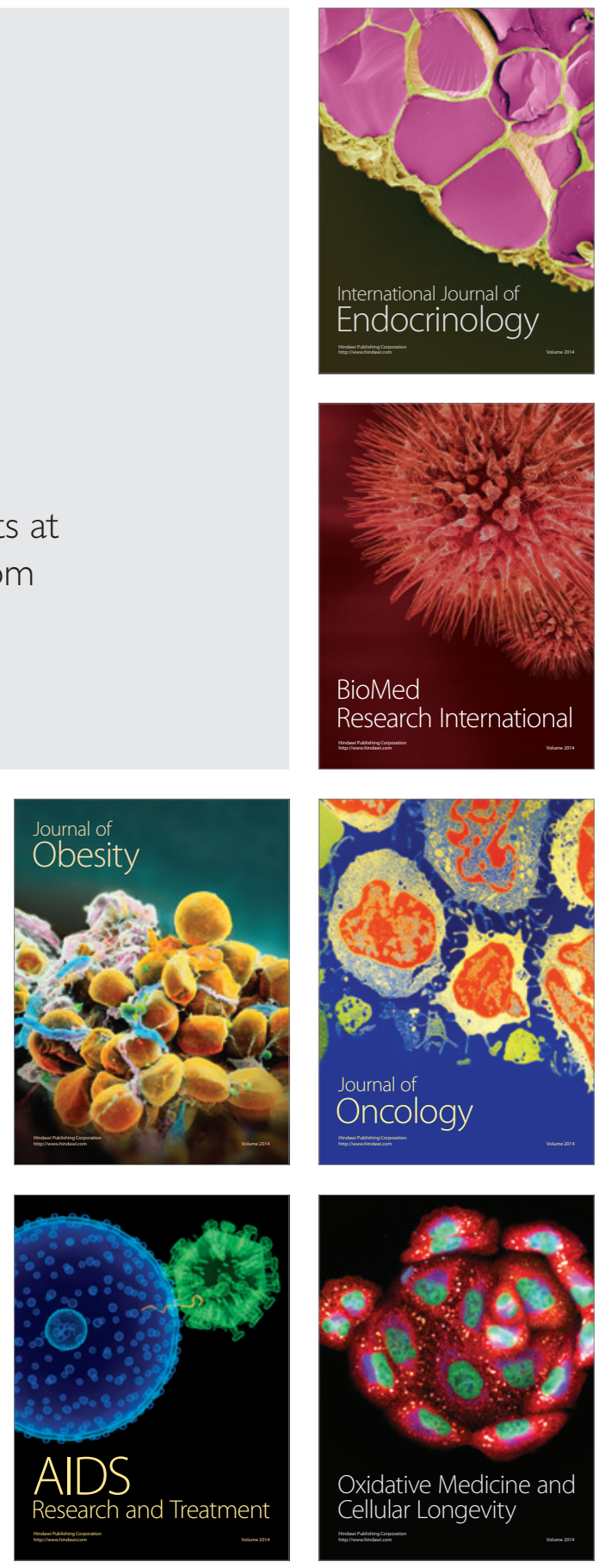